\title{
ON THE DUAL OF HORNICH'S SPACE
}

JOSEPH A. CIMA

Let $D$ be unit disk and $\mathcal{H}$ the set of analytic functions $f$ in $D$ with $f(0)=0, f^{\prime}(z) \neq 0$ and $\left|\arg f^{\prime}(z)\right| \leqq K=K_{f}, f^{\prime}(0)=1$. Hornich has defined operations on $\mathcal{F C}^{\mathrm{C}}$ so that it is a real Banach space [2]. That is if $f, g$ are in $\mathcal{H C}$ and $\alpha$ is real

$$
\begin{aligned}
{[f, g](z) } & =\int_{0}^{z} f^{\prime}(t) g^{\prime}(t) d t \\
{[\alpha \times f](z) } & =\int_{0}^{z}\left[f^{\prime}(t)\right]^{\alpha} d t
\end{aligned}
$$

and $\|f\|=\sup _{z^{\prime}, z^{\prime \prime} \in D}\left|\arg f^{\prime}\left(z^{\prime}\right)-\arg f^{\prime}\left(z^{\prime \prime}\right)\right|$. Let $\nu$ be a real valued, bounded, finitely additive set function on the Lebesgue measurable subsets of $|z|=1$ with $\nu(E)=0$ if $E$ is a Lebsegue null set. We will call $\nu$ a charge.

Theorem. The dual of the Hornich space on $D$ can be identified isomorphically with equivalence classes of charges on the unit circle.

Proof. Let $u_{0}$ be the linear space of bounded harmonic functions on $D$ vanishing at the origin. We define a map $T$ from $\mathcal{H C}$ to $\mathcal{U}_{0}$ by $T(f)=\arg f^{\prime}$. The normalization of functions in $\mathfrak{H C}$ and the CauchyRiemann equations guarantee that $T$ is one to one and onto $\mathcal{u}_{0}$. Moreover, if $\|u\|_{\infty}$ is the sup norm for a function $u \in \mathcal{u}_{0}$ we see that

$$
\left\|u_{f}\right\|_{\infty} \leqq\|f\|_{r c} \leqq 2\left\|u_{f}\right\|_{\infty}
$$

where $u_{f}$ is the image of $f$ under $T$. Thus $T$ is a bicontinuous map of $\mathcal{H}$ on to $u_{0}$. The Fatou theorem implies that $\lim _{r \rightarrow 1} u\left(r e^{i \theta}\right)=u(\theta)$ exists a.e. Also it can be shown that the essential sup norm of $u(\theta)$ coincides with the sup norm of $u$ in the disk. Further, if $\bar{u}$ is the equivalence class of $u(\theta)$ in $L^{\infty}$ then it is uniquely determined by $u$. The dual of $L^{\infty}$ is given by the charges $\nu$ on $|z|=1$, see N. Dunford and J. Schwartz [1, pp. 296-297]. Since $\mathcal{U}_{0}$ (regarded as a subspace of $L^{\infty}$ ) has codimension one we have the result. That is a functional on $\mathcal{H C}$ is given by an equivalence class $[\nu]$ of charges, where $\nu_{1} \sim \nu_{2}$ if and only if $\nu_{1}=\nu_{2}+k d \theta, k$ a constant.

We observe that the obvious linear functionals 1968.

Received by the editors September 11, 1968 and, in revised form, October 28, 


$$
T_{z_{0}}(f)=\arg f^{\prime}\left(z_{0}\right), \quad\left|z_{0}\right|<1
$$

are given by integrating the boundary values of arg $f^{\prime}$ against the Poisson kernel at $z_{0}$. Also one can show by examples that the evaluation maps $f \rightarrow f\left(z_{0}\right)$ are not continuous in the Hornich topology.

\section{REFERENCES}

1. N. Dunford and J. Schwartz, Linear operators. Part I, Interscience, New York, 1958.

2. H. Hornich, Ein Banachraum analytischer Funktionen in Zusammenhang mit den schlichten Funktionen, Monatsh. Math. 73 (1969), 36-45.

University of North Carolina 